\title{
Anatomía topográfica del asterion
}

\author{
F. Martínez****; A. Laxague*; L. Vida*; H. Prinzo, ${ }^{*}{ }^{* *}$; N. Sgarbi**; V.R Soria* y C. Bianchí***.
}

Departamento de Anatomía Humana Normal*. Facultad de Medicina. Departamento de Neurocirugía**. Hospital de Clínicas Dr, Manuel Quintela. Facultad de Medicina, Servicio de Otorrinolaringlogía***. Hospital Pereira Rossell. Montevideo. Uruguay

\section{Resumen}

Introducción. Las referencias anatómicas que permiten ubicar los senos venosos durante los abordajes de fosa posterior, son de utilidad para el neurocirujano. EI asterion es usado como referencia para localizar cl seno transverso (ST).

Material y método. Se utilizaron 50 hemicráneos de cadáveres adultos. Se estudió la relación del asterion con el ST, la vena emisaria mastoidea (VEM), la cresta suprameatal y el inion.

Resultados. Se identificó el asterion en 49 casos. En el $\mathbf{8 7 . 8 \%}$ de las piezas, se situó a la altura del seno transverso. El $\mathbf{7 2 . 2 \%}$ se ubicó sobre el propio seno y el $\mathbf{2 7 . 8 \%}$ sobre su codo. Si se traza una línea desde la cresta suprameatal al inion, el asterion se ubica por debajo de esta línea en el $88 \%$ de los casos.

El foramen de la VEM se identificó en 46 oportunidades, en el $36 \%$ fue doble.

Discusión y conclusiones. Según nuestros datos y los de otros autores, el orificio de trépano debe situarse por debajo y algo detrás del asterion para evitar la lesión del seno transverso.

PALABRAS CLAVE: Asterion. Fosa posterior. Seno transverso. Seno lateral. Vena emisaria mastoidea.

\section{Topographyc anatomy of the asterion}

\section{Abstract}

Background. In the surgical approaches to the posterior fossa, the accurate location of the transverse-sigmoid sinus (TS-SS) complex is of great importance. The asterion is a referal landmark to the transverse sinus location.

Method. Twenty-Iive skulls of adult cadavers were studied. We seek for the relationships of the asterion with: TS location, mastoid emissary vein, suprameatal

Recibido: 20-04-04. Aceptado: 23-06-04 crest and inion.

Results. The asterion was found in 49 cases. In the great majority of cases $(\mathbf{8 7 . 8 \%})$ the asterion was over the TS $(72.2 \%$ over the sinus proper, $27.8 \%$ over the TS-SS transition).

The mastoid emissary vein was present in 46 cases, and in $36 \%$ we found two veins.

Discussion and conclusions. The burr hole for posterolateral approaches to the posterior fossa must be located below and behind the asterion.

KEY WORDS: Asterion. Posterior fossa. Transverse sinus. Lateral sinus. Mastoid emissary vein.

\section{Introducción}

La fosa posterior está enmarcada por importantes estructuras venosas, como los senos laterales, petrosos superiores, occipital y la prensa de Herófilo. Durante los abordajes de la fosa posterior, el cirujano debe tener en cuenta la ubicación de los senos venosos para no lesionarlos. Por ello, es imprescindible contar con puntos de referencia anatómicos superficiales que permitan ubicar dichas estructuras, para hacer los orificios de trépano en un sitio seguro $^{1-9}$. Esto adquiere aún mayor importancia si se quiere llegar al blanco quirúrgico a través de abordajes pequeños.

Los puntos anatómicos utilizados como referencia para topografíar el seno lateral y la prensa de Herófilo son: la protuberancia occipital externa (POE), cresta suprameatal (CSM), apófisis mastoides, asterion, inserción del músculo esplenio capitis y línea nucal superior1-9.

Algunos de estos puntos de referencia pueden ser identificados mediante reconstrucción de imágenes y sistemas de neuronavegación, lo que permite planificar el abordaje previamente $^{9}$. Sin embargo, la mayoría de los centros neuroquirúrgicos no tienen acceso a estas técnicas, por lo que las referencias anatómicos continúan teniendo gran

Abreviaturas. POE: protuberancia occipital interna. CSM: cresta suprameatal. VEM: vena emisaria mastoidea. SL: seno lateral. S seno transverso. SS: seno sigmoides. 


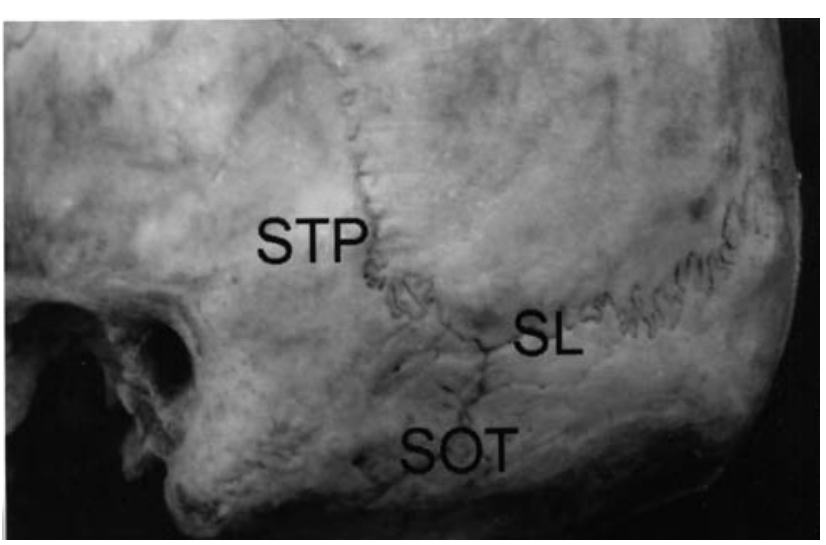

Figura 1 a: Vista posterolateral de la base del cráneo, lado izquierdo. Las suturas lambdoidea (SI.), occipito-temporal (SOT) y temporo-parietal (STP) convergen hacia el asterion. 1 b) Vista posterolateral de la base del cráneo, lado derecho. Se muestran las medidas tomadas desde el asterion hacia: a) protuberancia occipital externa, b) resta suprameatal, c) vena emisaria mastoidea, d) vértice de la apófisis mastoides.

valor $^{4,6,7}$.

El asterion (del griego, estrella), es el sitio de unión de las suturas lambdoidea, occipitomastoidea y temporoparietal $^{2,10,11}$. Este punto puede ser reconocido durante los abordajes posterolaterales a la fosa posterior y es usado para ubicar la porción transversa del seno lateral (seno transverso) y su codo ${ }^{2,6}$. Sin embargo, para algunos autores, las relaciones del asterion con el seno transverso (ST) son variables, lo que limitaría su valor ${ }^{2-4,6,7}$.

El presente estudio anatomodescriptivo, se centra en las relaciones del asterion con las estructuras vasculares y óseas de importancia en los abordajes posterolaterales a la fosa posterior.

\section{Material y método}

Se estudiaron 50 hemicráneos de cadáveres adultos.

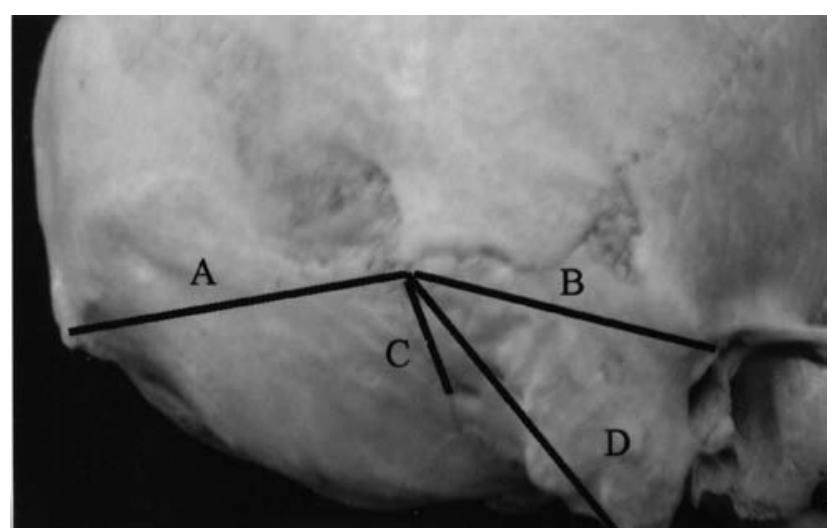

Del total, 45 fueron cráneos secos y 5 cráneos frescos sin encéfalo.

En cada preparado se tomaron las medidas desde el asterion a las siguientes estructuras (figura 1): a) protuberancia occipital externa (POE); b) cresta suprameatal (CSM); e) vena emisaria mastoidea (VI:M); d) vértice de la apófisis mastoides.

Se registró, además: el número de VEM, la situación del asterion en relación con una línea que une la CSM con la POE y la topografía del seno lateral (SL) con respecto al asterion. Esto último se corroboró colocando un compás con una rama sobre el asterion y otra sobre el endocráneo. El compás fue situado perpendicular a la escarpa del occipital.

En las piezas secas, la VEM se identificó a partir de los forámenes vasculares ubicados en la topografía habitual de dicha vena.

Tabla 1

Distancias medias desde el asterion a los puntos de referencia

\begin{tabular}{|l|c|c|c|}
\hline Medida & Rango $(\mathrm{mm})$ & Promedio $(\mathrm{mm})$ & Mediana $(\mathrm{mm})$ \\
\hline $\begin{array}{l}\text { Asterion- } \\
\text { POE }\end{array}$ & $49-86$ & 64.4 & 65 \\
\hline $\begin{array}{l}\text { Asterion- } \\
\text { CSM }\end{array}$ & $31-55$ & 44.9 & 45.5 \\
\hline $\begin{array}{l}\text { Asterion- } \\
\text { VEM }\end{array}$ & $8-29$ & 19.5 & 19 \\
\hline $\begin{array}{l}\text { Asterion- } \\
\text { Mastoides }\end{array}$ & $38-56$ & 49.7 & 50 \\
\hline
\end{tabular}

POE: protuberancia occipital externa. CSM: cresta suprameatal, VEM: vena emisaria mastoidea. 


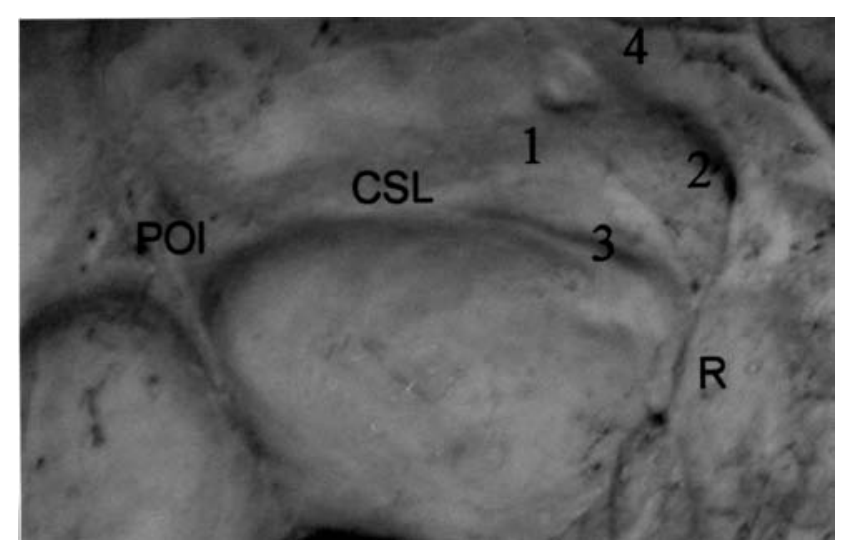

Figura 2. Vista de la topografía del trayecto el seno lateral desde el endocráneo, lado izquierdo. Se observan la protuberancia occipital interna (POI), el canal del seno lateral (CSL) en la escama del occipital y el peñasco del temporal (R). Con los números 1 a 4 se muestra la relación del asterion con: 1) porción transversa del seno lateral (21.4\%), 2) codo del seno (63.4\%), 3) y 4) asterion por abajo y arriba del seno lateral (9.8 y 2.4\% respectivamente).

\section{Resultados}

En un caso no se reconoció el asterion como tal. En dicha pieza, en el punto de unión de las tres suturas se ubicó un hueso wormiano. En este caso, el punto de referencia para tomar las medidas fue el sitio en donde las tres suturas se cruzarían luego de prolongarlas según su eje.

Los rangos y promedios de los valores hallados para las medidas a, b, c y d se muestran en la tabla 1. El modo para cada una de estas medidas fue: $65,47,25$ y $49 \mathrm{~mm}$ respectivamente.

La ubicación del asterion con respecto al seno lateral se muestra en la figura 2. Como se pone en evidencia, en la mayor parte de los casos, el asterion está situado a la altura del ST. Del total de los casos en donde el asterion se ubicó a nivel del ST, el 72.2\% se situó sobre el propio seno, en tanto en el $27.8 \%$ lo hizo sobre su codo.

Se encontró la vena emisaria mastoidea en el 94\% de los casos; el 36\% de las piezas presentaron dos venas. La topografía habitual de la o las VEM fue a nivel de la sutura occipitomastoidea o a pocos milímetros de la misma. Si se divide la distancia asterion-vértice de la apófisis mastoides en tres tercios, en el 17\% de los casos, la VE.M se ubicó en el tercio proximal. En el $70 \%$ se situó en el tercio medio y en el $13 \%$ en la unión de ambos.

\section{Discusión}

En los abordajes posterolaterales a la fosa posterior, es fundamental tener en cuenta la topografía superficial de los senos venosos a fin de evitar su lesión ${ }^{2}$. La misma puede
2005; 16: 441-446

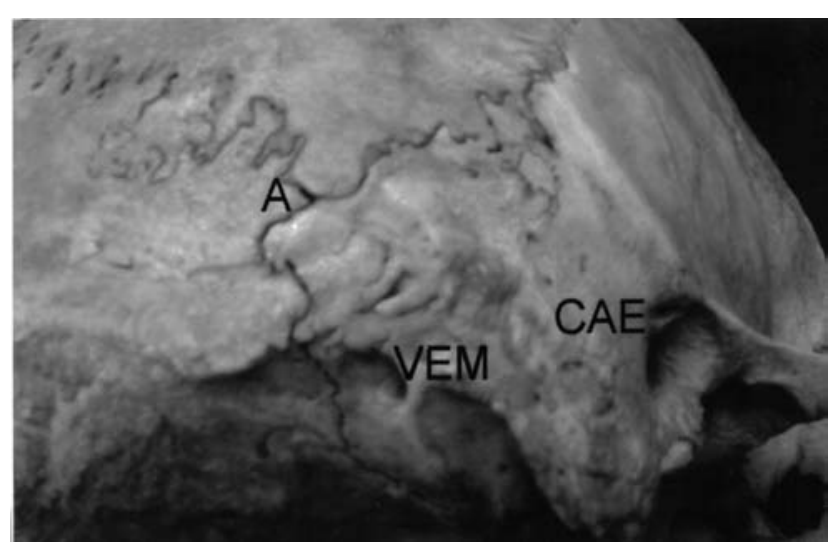

Figura 3a. Vista posterolateral de la base del cráneo, lado derecho. Cerca de la base de la apófisis mastoides, se observa un voluminoso orificio correspondiente al punto de salida de la vena emisaria mastoidea (VGM).

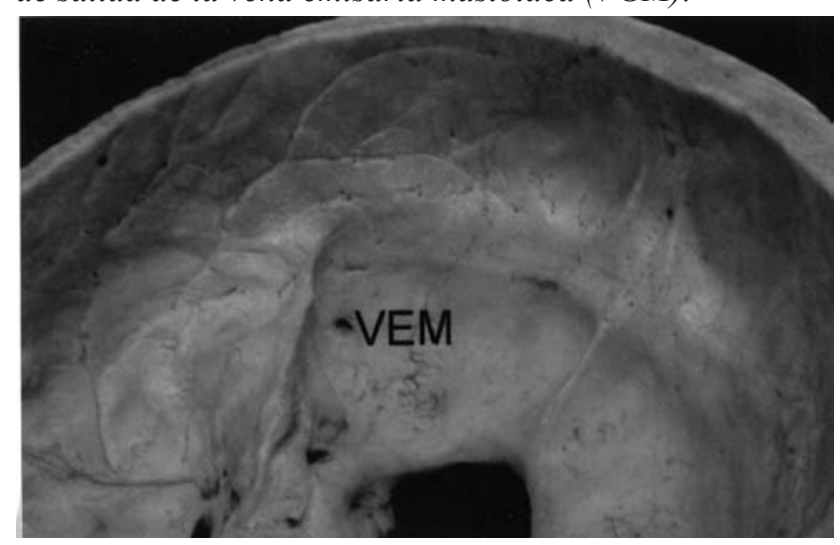

Figura 3b. Vista endocraneana del mismo ejemplar.

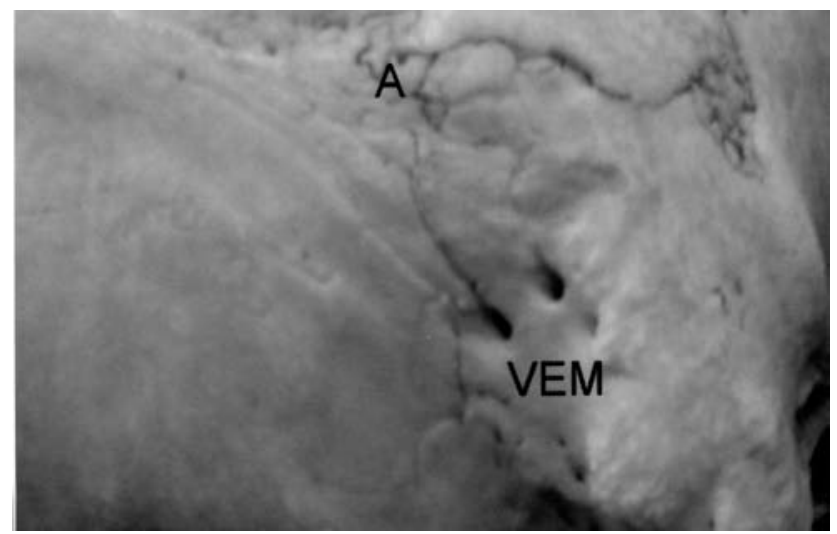

Figura 3c. Vista posterolateral de la base del cráneo, lado derecho. Vena emisaria doble.

tener graves consecuencias, en particular el sangrado o la embolia aérea. Además de los senos venosos, puede haber embolia aérea o sangrado a partir de venas musculares o emisarias $1,2,8,12,13$.

Por ello, es sumamente útil para el neurocirujano conocer con exactitud la ubicación de dichas estructuras y, en 
particular, su relación con los reperes óseos utilizados en estos abordajes.

Varios autores se han ocupado de buscar referencias topográficas para ubicar las diferentes porciones del seno lateral. Este importante seno venoso se origina en la prensa de Herófilo y se dirige hacia fuera y algo abajo en el surco lateral del occipital. Luego se incurva hacia abajo y afuera para descender en el canal petromastoideo, en relación con el sector posterior de la mastoides. Llega al foramen yugular, donde se continúa con el bulbo de la vena yugular interna. Esto permite reconocer tres porciones en el seno lateral: una horizontal (seno transverso), un codo o rodilla y otra descendente (seno sigmoides) $)^{2,4,7,14-20}$.

\section{Topografía del seno lateral y sus porciones}

Generalmente se acepta que la prensa de Herófilo se ubica entre 5 y $10 \mathrm{~mm}$ por arriba de la $\mathrm{POE}^{4-6}$.

El ST se sitúa en la circunferencia externa del tentorio, en una línea tendida desde la CSM hasta la $\mathrm{POE}^{2,14,19,20}$. El seno sigmoides se sitúa en una línea que parte del vértice de la mastoides y sigue su eje hacia arriba y atrás llegando al asterion. En el punto de cruce de estas dos líneas se encuentra el codo del seno lateral ${ }^{2}$.

Estas relaciones pierden su valor en pacientes que presentan alteraciones del desarrollo en la fosa posterior, como ocurre en las malformaciones de Chiari y DandyWalker ${ }^{5,21-26}$. Normalmente la tienda del cerebelo sufre una migración caudal por el desarrollo del cerebro, y se sitúa en su posición definitiva al tercer mes de vida intrauterina ${ }^{21}$. Las alteraciones de esta migración hacen variar la posición final de la tienda del cerebelo y por lo tanto la situación de los senos en relación con la misma.

En la malformación de Chiari, hay una herniación del cerebelo a través del foramen magno, lo que permite que la tienda del cerebelo descienda más de lo habitual. Por ello, el tentorio es mas vertical y mas bajo, al igual que el propio seno lateral ${ }^{23-25}$.

Lo contrario ocurre en la malformación de DandyWalker, donde la presencia de quistes vermianos produce desplazamientos y atrofia del cerebelo. El quiste vermiano produce generalmente un aumento del volumen de la fosa posterior, que impide el normal descenso de la tienda y aumenta el tamaño del foramen de Pacchioni. En estos pacientes, el seno lateral se localiza por arriba de su situación normal ${ }^{22,26}$.

\section{Relaciones del asterion con el seno transverso}

El asterion se encuentra por detrás y arriba de la mastoides y, en profundidad, se corresponde con el borde inferior del codo del seno lateral ${ }^{6}$. Por ello, en los abordajes posterolaterales a la fosa posterior, algunos neurocirujanos plantean que el orificio de trépano debe ubicarse por debajo y detrás del asterion, alejándose del seno $3,6,7,18$.

Varios estudios anatómicos en cadáveres frescos y cráneos secos evidencian que las relaciones del asterion con el seno transverso son variables y algo diferentes a lo que clásicamente se describe ${ }^{2-4,6,18}$.

Uz y cols. ${ }^{7}$ encuentran que en el $54 \%$ de los casos, el asterion se sitúa a nivel del seno lateral y en un $44 \%$ lo hace por debajo, en la duramadre de la fosa posterior. Para Díaz Day y cols., ${ }^{3,18}$ entre el 55 y el 61\%, de los casos el asterion se ubica a nivel del seno. Para Sripairjkul y Adultrakoon, el asterion se ubica sobre el seno lateral entre el $58.1 \mathrm{y}$ el $74.4 \%$ (a derecha e izquierda respectivamente). Nuestros hallazgos son coincidentes con estos trabajos, aunque el porcentaje de casos donde el asterion está a la altura del seno (ya sea el propio seno o su codo) son algo mayores $(87.8 \%)$.

Para Rhoton 6 , el asterion se sitúa en relación con la mitad inferior del codo del SL. En este estudio, del total de los casos que se situaron a la altura del seno, sólo el $27.8 \%$ se ubicó cerca de la rodilla y el $72.2 \%$ sobre el ST.

De acuerdo a nuestros hallazgos y los de la mayoría de los autores consultados, se desprende que un orificio de trépano situado 1 o $2 \mathrm{~cm}$. por debajo del asterion está alejado del seno lateral, ya sea el propio seno o el codo.

\section{Relaciones del asterion con otras referencias óseas}

Sobre las distancias medidas desde el asterion a los otros puntos óseos de referencia (CSM, vértice de la apófisis mastoides e inion), nuestros hallazgos no difieren de los de otros investigadores ${ }^{3,4}$.

Por ejemplo, la distancia asterion-vértice de la apófisis mastoides fue en promedio $50.3 \mathrm{~mm}$ para Avci et $\mathrm{al}^{1}, 49.2 \mathrm{~mm}$ para Díaz Day et al3 y $49.7 \mathrm{~mm}$ en nuestros casos.

La distancia CSM-asterion fue en promedio $41.4 \mathrm{~mm}$ para Díaz Day et al. ${ }^{3} 44.8 \mathrm{~mm}$ en el presente estudio.

\section{Vena emisaria mastoidea}

Esta vena es muy constante y comunica la circulación intra y extracraneana. En particular, anastomosa el seno lateral con las venas occipitales y cervicales profundas ${ }^{12,14-}$ ${ }^{16}$. El calibre de esta vena puede ser importante y en el caso de tumores de crecimiento lento que obstruyan o compriman los senos venosos (como los meningiomas), actúa como una importante vía de circulación colateral ${ }^{15,27}$. El conocimiento de la situación de esta vena tiene gran importancia, ya que posee un trayecto transeraneano no colapsable. Esto hace que la VEM sea una fuente potencial de sangrado o embolia aérea ${ }^{1,2,12,27}$. De esta forma, el cirujano deberá anticipar su presencia para coagularla y seccionarla 
en el sector extracraneano, o colocar cera hemostática en su salida del cráneo.

En este estudio, la VEM fue muy constante (94\% de los casos), como es descrito por varios autores ${ }^{8,15,20}$. Yasargil ${ }^{27}$ menciona que pueden verse una o dos VEM; esto último ocurrió en el 36\% de los casos en nuestra serie.

Sobre su topografía, Díaz Day $^{2}$ destaca que si se traza una línea que una el asterion con el vértice de la mastoides, generalmente la VEM se sitúa a mitad de camino entre ambos puntos. Esto es corroborado por nuestro estudio, ya que en el $70 \%$ de los casos la VEM se ubicó en el tercio medio de dicha línea.

\section{Conclusiones}

El conocimiento detallado de las relaciones superficiales de los senos venosos en relación con el tentorio es la base para evitar su lesión al abordar la fosa posterior.

El asterion se sitúa en la mayoría de los casos a la altura del seno transverso, por lo tanto, los orificios de trépano deberían situarse alejados de este repere.

La vena emisaria mastoidea es muy constante y en mas de un tercio de los casos es doble. Lo mas frecuente es que se ubique en la sutura petromastoidea, a mitad de camino entre el asterion y el vértice de la apófisis mastoides.

\section{Agradecimientos:}

Uno de los autores (FM) quiere agradecer a las siguientes personas su colaboración en este trabajo:

Ass. Prof. Haber Feraz-Leite, Departamento de Neurocirugía del Hospital Universitario de Viena, Austria. Dr. Mario Gallarreta y Pte. Int. Rodrígo Moragues, Servicio de Neurocirugía del 1lospital Maciel, Montevideo, Uruguay.

\section{Bibliografía}

1. Avci, E., Kocaogullar, Y., Fossett, D., Caputy, A.: Lateral posterior fossa venous sinus relationships to surface landmarks. Surg Neurol 2003; 59: 392-397.

2. Babcock, D.S., Han, B.K.: Ultrasongraphy of the head. En Section of Pediatric Neurosurgery of AANS (eds). Pediatric Neurosurgery. Surgery of the developing nervous system. Grune \& Stratton, New York, 1982; pp. 707-720

3. Bracard, S., Braun, M., Meder, J.F., Velut, S.: Anatomie et radioanatomie du systeme veineux intracraniene (á I'exclusion des sinus duraux). Neurochirurgie 1996; 42 (Suppl 1): s11 -s44.

4. Carmel, P.W.: The Arnold-Chiari malformation. En Section of Pediatric Neurosurgery of AANS (eds). Pediatric Neurosurgery. Surgery of the developing nervous system. Grune \& Stratton, New York, 1982; pp. 61-78.

5. Davis, G.: Anatomia Applicata. Societá Editrice Libra- ria, Milan, 1913;pp. 20-29.

6. Detwiler, P.W., Porter, R.W., Rekate, H.L.: Hydrocephalus-Clinical features and management. En Choux, M., Di Rocco, C., Hockley, A.D., Walker, M.L., (eds). Pediatric Neurosurgery. Churchill Livingstone, London, 1999; pp. 253-274

7. Dias, M.S.: Myelomeningocele. En Choux, M., Di Rocco, C., Ilockley, A.D., Walker, M.L. (eds). Pediatric Neurosurgery. Churchill Livingstone, London, 1999; pp. 33-60.

8. Díaz Day, J., Kellogg JX Tschabitscher, M., Fukushima, T.: Surface and superficial surgical anatomy of the posterolateral cranial base: significance for surgical planning and approach surgical anatomy. Neurosurgery 1996; 38: 10791084.

9. Díaz Day, J., Tschabitscher, M.: Microsurgical dissection of the cranial base. Churchill Livingstone, New York, 1996.

10. Díaz Day, J., Tschabitscher, M.: Anatomic position of the asterion. Neurosurgery 1998; 42: 198-199.

11. French, B.N.: Midline fusion defects and defects of formation. En Youmans, J.R. (ed). Neurological surgery. $2^{\text {nd }}$, ed, Vol. 3. WB Saunders Company, New York, 1982; pp. 1236-1381.

12. Gusmao, S., Leal Silveira, R., Arantes, A.: Pontos referenciais nos acessos cranianos. Arq Neuropsiquiatr 2003; 61: 305-308.

13. Krause, F.: Chirurgie du cerveau et de la moelle épiniére. Société d'éditions scienti(iques et médicales, Paris, 1912; pp. 21-32.

14. Lippert, H.: Anatomía. Estructura y morfología del cuerpo humano. $4^{\mathrm{a}}$ ed, Marban, Madrid, 2003; pp. 459-462.

15. Poirier, P.: Traité D'Anatomie Humaine. $2^{\circ}$ ed, Masson, Paris, 1896 ;pp. 955-987.

16. Rhoton, A.I,. Jr.: The far-lateral approach and its transcondylar, supracondylar and paracondylar extensions. Neurosurgery 2000; 47(3) suppl 1: s195-s210.

17) Rouviere, H., Delmás, A.: Anatomía humana. Descriptiva, topográfica y funcional. $10^{\mathrm{a}} \mathrm{ed}$, tomo I. Masson, Barcelona, 2001; pp. 208-224.

18) Sindou, M., Alaywan, M., Allacq, P.. Les sinus duraux. A: Chirurgie des grands sinus veineux duraux intraerániens. Neurochirurgie 1996; 42 (Suppl 1): s42-s87.

19) Sripairojku1, B., Adultrakoon, A.: Anatomical position of the asterion and its underlying structure. J Med Assoc Thai. 2000 Sep; 83:1112-1115 (Abstract).

20) Tarlov, F.: Surgical management of tumors of the tentorium and clivus. En Schmidek, H.H., Sweet, W.H. (eds). Operative neurosurgical techniques. Grune \& Stratton, New York, 1982; pp. 381-388.

21) Testut, L.: Tratado de anatomía humana. $8^{\mathrm{a}}$ ed, Tomo 2. Salvat, Barcelona, 1943; pp. 433-461.

22) Tew, J.M. Jr., van Loveren, H.R., Keller, JT.: Atlas of operativa neurosurgery. WB Saunders Company, Philadelphia, 
2001; pp. 154-177.

23) Tillaux, P.: Traité D'Anatomie topographique avec applications a la chirurgie. $9^{\mathrm{eme}} \mathrm{ed}$, Asselin et Houzeau, Paris, 1879; pp. 28-52.

24) Tubbs, R.S., Salter, G., Oakes, W.J.: Superficial surgical landmarks for the transversa sinus and torcular herophili. J Neurosurg 2000; 93: 279-281.

25) Uz, A., Ugur, H.C., Tekdemir, I.: Is the asterion a reliable landmark for the lateral approach to posterior fosa?. J Clin Neurosci 2001; 8: 146-147.

26) Vrionis, F.D., Foley, K.T., Robertson, J.H.., Shea, J.J. III.: Use of cranial surface anatomic fiducials for interactive image-guided navigation in the temporal bone: a cadaveric study. Neurosurgery 1997; 40:755-764.

27) Yasargil, M.G.: Microneurosurgery. Vol IV B, Microsurgery of CNS tumors. George Thieme Verlag, Stuttgart, 1996: pp. 58-64, 100-123.

Martínez, F.; Laxague, A,; Vida, L.; Prinzo, H.;. Sgarbi, N.; Soria, V.R.; Bianchí, C.: Anatomía topográfica del asterion. Neurocirugía 2005; 16: 441-446.

Correspondencia postal: Dr. Fernando Martínez. Departamento de Anatomía Humana Normal, Facultad de Medicina. Avda. Gral. Flores 2125, esquina Yatay, Montevideo, Uruguay. 